PSYCHOMETRIKA-VOL. 17 , NO. 2

JUNE, 1952

\title{
ON THE DETERMINATION OF REDUNDANCIES IN SOCIOMETRIC CHAINS*
}

\author{
Ian C. Ross and Frank Harary \\ UNIVERSITY OF MICHIGAN
}

\begin{abstract}
The use of a matrix to represent a relationship between the members of a group is well known in sociometry. If this matrix is raised to a certain power, the elements appearing give the total number of connecting paths between each pair of members. In general, some of these paths will be redundant. Methods of finding the number of such redundant paths have been developed for three- and four-step chains by Luce and Perry (3) and Katz (2), respectively. We have derived formulas for the number of redundant paths of five and six steps; and in addition, an algorithm for determining the number of redundant paths of any given length.
\end{abstract}

\section{Introduction}

The problem of redundant paths in communication matrices has been open for some time. Solutions for the third and fourth powers of the matrix have been published by Luce and Perry (3) and Katz (2).

A method is presented by which the number of redundant paths in any power of the matrix may be calculated. By this method, the matrix of redundant paths is expressed in terms of the given matrix and some matrix operations. In addition to applying this procedure to three- and fourstep redundant paths, we have derived formulas for the cases of five- and six-step redundant paths. The method is an elementary application of a wellknown identity involving combinations to a partition function which is appropriate for this problem. Numerical examples are given to illustrate the use of each formula.

\section{Redundant Paths and Partitions}

2.1. Redundant Paths. In a group of $n$ people (denoted by the letters $i, j, k, \cdots)$ a one-slep path exists between $i$ and $j$ if $i$ communicates with $j$. A path from $i$ to $j$ is a sequence of steps beginning with $i$ and ending with $j$, $i \neq j$. For example, $i k j$ is a two-step path from $i$ to $j$; $i k l k j$ is a four-step path from $i$ to $j$; etc. A redundant path is a path in which at least one letter (person) occurs more than once, as in the paths $i k l k j, i k i j$, ijijij, and $i k l k l j$. Let the $n \times n$ matrix $M$ represent the communication pattern of the particular group under consideration, in which the $i, j$ entry is 1 if $i$ communicates Foundation.

*The research leading to this paper was supported by a grant from the Rockefeller 
with $j$ and 0 otherwise. It is assumed that no individual communicates with himself and therefore the main diagonal of the matrix consists only of zeros. As pointed out by Festinger (1), the sth power of the matrix $M$ has as its $i, j$ entry the total number of $s$-step paths (both redundant and nonredundant) from $i$ to $j$.

Let $R$, denote the matrix whose $i, j$ entry is the number of redundant $s$-step paths from $i$ to $j$. Obviously, the $i, j$ entry of $R_{*}$ for $i \neq j$ is a nonnegative integer which is not greater than the corresponding entry of $M^{*}$. It is also obvious that $R_{*}=M^{*}$ except possibly on the main diagonal for all $s \geq n$ since any path involving more than $n-1$ steps and drawn from $n$ letters, must contain at least one letter more than once.

2.2. Partitions. A particular kind of partition is useful in the consideration of redundant paths; namely, the number of ways in which the positive integer $s$ can be written as the sum of three integers, such that the first and third are non-negative but not both zero, the second is greater than one, and the order of the summands is significant. Let $s=s_{1}+s_{2}+s_{3}$ be such a partition of $s$. A redundant path from $i$ to $j$ is said to satisfy this partition if the number of steps from the initial $i$ to any of the places in which a repeated letter occurs other than its last appearance, is $s_{1}$; the number of steps from this appearance of the repeated letter to a later appearance is $s_{2}$; and the number of steps from this second appearance to the terminal $j$ is $s_{3}$. Thus all the partitions of 3 are:

$$
\begin{aligned}
& 3=0+2+1, \\
& 3=1+2+0 .
\end{aligned}
$$

For example, the redundant path $i k i j$ satisfies the partition $0+2+1$ in that the number of steps from the first letter to the repeated letter is 0 , the number of steps from the repeated letter to its second appearance is 2 , and the number of remaining steps to the last letter is 1.

Some redundant paths satisfy more than one partition. Thus $i j i j$ satisfies $0+2+1$ when $i$ is considered as the repeated letter and $1+2+0$ when $j$ is so considered. Also two partitions of $s$ may be inconsistent in the sense that there is no path satisfying both of them.

We conclude this section with two obvious remarks regarding these partitions which will be used below.

Remark 1: The maximum number of partitions which are satisfied by a redundant $s$-step path is*

$$
2 C\left(\left[\frac{s+1}{2}\right], 2\right)
$$

*The notation $[x]$ used in the following formula is defined as usual by: $[x]=$ the largest integer which is not greater than $x$. Also $C(n, r)$ denotes the number of combinations of $n$ objects taken $r$ at a time. 
Proof: If $s$ is odd, the redundant path with the greatest number of partitions is $i j i j \cdots i j$. The number of partitions in which $i$ is considered as the repeated letter is $C\left(\frac{s+1}{2}, 2\right)$ since there are $s+1$ letters (including multiplicity) half of which are $i$ and these are combined two at a time. Obviously the corresponding number of partitions considering $j$ as repeated is the same. Hence the maximum number is $2 C\left(\frac{s+1}{2}, 2\right)$ when $s$ is odd.

If $s$ is even, each redundant path with the greatest number of partitions is of the form $i j i j \cdots k \cdots i j$ with exactly one letter $k$. In such a path, $s / 2$ of the letters are $i$ and $s / 2$ are $j$. Hence the maximum number of partitions is as above $2 C(s / 2,2)$. But when $s$ is even, $\frac{s}{2}=\left[\frac{s+1}{2}\right]$.

Therefore regardless of the parity of $s$, the maximum number of partitions is $2 C\left(\left[\frac{s+1}{2}\right], 2\right)$.

Remark 2: The number of partitions of $s$ is $C(s, 2)-1$.

Proof: Clearly the number of partitions of $s$ as defined above is the number of partitions of $s$ or less into two summands such that the first is non-negative and the second is greater than one but less than $s$, in which the order of summands is significant. For $0+s+0$ is not a partition of $s$ according to our definition.

When the second summand is $s-1$ there are two possible first summands, namely 0 and 1 . As the second summand decreases by one, the number of possible first summands increases by one, etc. Therefore the number of partitions of $s$ is $2+\cdots+(s-1)$ which is well known to equal $C(s, 2)-1$.

\section{Procedure for Counting Each Redundant Path Exactly Once}

We wish to find for each $i, j$ and each $s$ the number of redundant paths from $i$ to $j$. The number of redundant $s$-step paths from $i$ to $j$ satisfying each partition of $s$ may be readily expressed in terms of the given matrix. However, the sum of these partitions may be more than the total number of redundant paths since a path may satisfy more than one partition. This difficulty can be handled by the use of the following identity involving combinations:

$$
\begin{aligned}
1 & =C(r, 1)-C(r, 2)+C(r, 3)-C(r, 4)+\cdots+(-1)^{r+1} C(r, r) \\
& =\sum_{k=1}^{r}(-1)^{k+1} C(r, k),
\end{aligned}
$$

whose validity for any positive integer $r$ is implied at once by the binomial theorem and the fact that $(1-1)^{r}=0$. 
For if the total number of redundant paths from $i$ to $j$ is $W_{i i}$, the value of $W_{i}$ can be determined as follows.

Let (i) $f_{1}, f_{2}, \cdots, f_{\mathrm{q}}$ be the set of all partitions of $s(q=C(s, 2)-1$ by Remark 2 ),

(ii) $a_{x}$ be the number of paths satisfying $f_{x}$,

(iii) $A_{1}=\sum_{x=1}^{a} a_{x}$,

(iv) $a_{x, y}$ be the number of paths satisfying both $f_{x}$ and $f_{y}, x \neq y$,

(v) $A_{2}=\sum_{\substack{x, y=1 \\ x<y}}^{q} a_{x, y}$,

and in general

(vi) $a_{x_{1}, x_{2}, \cdots, x_{t}}$ be the number of paths simultaneously satisfying all the partitions $f_{x_{1}}, f_{x_{2}}, \cdots, f_{x_{1}}$, and

(vii) $A_{t}=\sum_{x_{1}<x,<\cdots<x_{t}} a_{x_{i}, x_{2}, \cdots, x_{t}}$

for each $t$ from 1 to $u=2 C\left(\left[\frac{s+1}{2}\right], 2\right)$ (by Remark 1 ).

Then it follows from the combinatorial identity above that

$$
W_{i i}=A_{1}-A_{2}+A_{3}-\cdots+(-1)^{1+u} A_{u} .
$$

Let $R_{a}^{(1)}$ be the matrix whose $i, j$ entry is $A_{t}$. Then (1) can be expressed as a matrix equation which yields $R_{s}$, the matrix of redundant $s$-step paths whose $i, j$ entry is $W_{i i}$, namely:

$$
\begin{aligned}
R_{s} & =R_{s}^{(1)}-R_{s}^{(2)}+R_{0}^{(3)}-\cdots+(-1)^{1+u} R_{s}^{(u)} \\
& =\sum_{i=1}^{u}(-1)^{t+1} R_{s}^{(l)}
\end{aligned}
$$

\section{Redundant Paths of Three and Four Steps}

4.1. Notation. Throughout this subsection, let $A, B$ be $n \times n$ matrices whose $i, j$ entries are $a_{i i}, b_{i i}$, respectively.

This is also expressible by $A=\left\|a_{i i}\right\|, B=\left\|b_{i i}\right\|$.

The usual definitions of matrix addition, $A+B$; matrix subtraction, $A-B$; multiplication of matrix $A$ by scalar $c, c A$; ordinary multiplication of matrices, $A \cdot B$; and the transpose of matrix $A, A^{\prime}$, are assumed and are given in detail in Weiss (4). Two other operations will also be required:

(1) Elementwise matrix multiplication $\mathrm{A} \times \mathrm{B}$ : $^{*}$

$$
A \times B=\left\|a_{i i} b_{i j}\right\| \text {. }
$$

*Elementwise matrix multiplication is due to Hadamard, as mentioned in Paul $R$. Halmos, Finite Dimensional Vector Spaces, Princeton University Press, 1942. 
(2) The diagonal operator $\mathrm{d}(\mathrm{A})$ :

$d(A)$ is the matrix whose principal diagonal is that of $A$ and whose remaining entries are zeros.

Referring specifically now to the given communication matrix $M$, we introduce the notation $S=M \times M^{\prime}=$ the matrix of mutual communications in the group.

Using the notation just introduced, we shall next express $R$. in terms of the given matrix $M$ for $s=3$ and $s=4$.

4.2. Derivation of $R_{3}$. By equation (2), $R_{3}=R_{3}^{(1)}-R_{3}^{(2)}$.

The admissible partitions of 3 are $f_{1}: 1+2+0$, and $f_{2}: 0+2+1$. The matrices of all 3-step paths satisfying $f_{1}$ and $f_{2}$ are $M \cdot d\left(M^{2}\right)$ and $d\left(M^{2}\right) \cdot M$, respectively. Therefore $R_{3}^{(1)}=M \cdot d\left(M^{2}\right)+d\left(M^{2}\right) \cdot M$.

The matrix of all 3-step paths satisfying both $f_{1}$ and $f_{2}$ is $M \times M^{\prime}$. Therefore $R_{3}^{(2)}=M \times M^{\prime}=S$. Hence

$$
R_{3}=\left[M \cdot d\left(M^{2}\right)+d\left(M^{2}\right) \cdot M\right]-S .
$$

This is the same formula as obtained by Luce and Perry (3), but the notation is different.

4.3. Derivation of $R_{4}$. By equation (2) again, $R_{4}=R_{4}^{(1)}-R_{4}^{(2)}$. The partitions of 4 are:

$f_{1}: 130$ (here 130 is an abbreviation of the partition $4=1+3+0$ ), $f_{2}: 031, f_{3}: 220, f_{4}: 022, f_{5}: 121$.

The following table lists these five partitions, with the most general path from $i$ to $j$ satisfying each and the matrix whose $i, j$ entry is the number of such paths.

TABLE 1

\begin{tabular}{|c|c|c|c|c|c|}
\hline $\begin{array}{l}\text { Partition } \\
\text { General Path } \\
\text { Matrix }\end{array}$ & $\begin{array}{c}f_{1} \\
i j k l j \\
M \cdot d\left(M^{3}\right)\end{array}$ & $\begin{array}{c}f_{2} \\
i k l i j \\
d\left(M^{3}\right) \cdot M\end{array}$ & $\begin{array}{c}f_{3} \\
i k j l j \\
M^{2} \cdot d\left(M^{2}\right)\end{array}$ & $\begin{array}{c}f_{1} \\
i k i l j \\
d\left(M^{2}\right) \cdot M^{2}\end{array}$ & $\begin{array}{c}f_{5} \\
i k l k j \\
M \cdot d\left(M^{2}\right) \cdot M\end{array}$ \\
\hline
\end{tabular}

We show how the matrix associated with $f_{1}$ is found. This matrix will bave as its $i, j$ entry the product $m_{i j} m_{i k} m_{k l} m_{l i}$ where $M=\left\|m_{i i}\right\|$ and each repeated index other than $i$ and $j$ is understood to be summed from 1 to $n$. But the matrix whose entries are $m_{i k} m_{k l} m_{l i}$ is $d\left(M^{3}\right)$. Therefore the desired matrix is $M \cdot d\left(M^{3}\right)$. The other matrices are derived similarly. Thus $R_{4}^{(1)}=\left[M \cdot d\left(M^{3}\right)+d\left(M^{3}\right) \cdot M\right]+\left[M^{2} \cdot d\left(M^{2}\right)+d\left(M^{2}\right) \cdot M^{2}\right]$ $+M \cdot d\left(M^{2}\right) \cdot M$. 
The consideration of the notion of inconsistent paths mentioned in Section 2.2 is now relevant. As previously stated, two partitions are called consistent if the existence of a path satisfying both is possible, and inconsistent otherwise. To illustrate consistency of two of the partitions of 4, consider $f_{1}$ and $f_{2}$. The most general paths satisfying $f_{1}$ and $f_{2}$ are $i j k l j$ and $i k l i j$, respectively. Hence the most general path satisfying both $f_{1}$ and $f_{2}$ is $i j k i j$ and such paths may exist.

To illustrate inconsistency consider $f_{1}$ and $f_{3}$. The most general path satisfying $f_{3}$ is $i k j l j$. Hence the most general path satisfying both $f_{1}$ and $f_{3}$ is ijjlj and no such path can exist since $d(M)=0$.

Another way in which two partitions may be inconsistent is illustrated by $f_{3}$ and $f_{4}$. A path satisfying both of these partitions would have to exhibit both $i$ and $j$ in exactly the same position in the path.

To find $R_{4}^{(2)}$ we construct a consistency table for these five partitions. In this table, 1 denotes consistency; 0 inconsistency.

Proceeding as in these illustrations, the consistency table is:

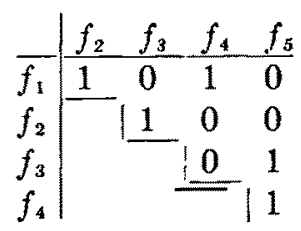

The following table lists those pairs of partitions which are consistent, with the most general path from $i$ to $j$ satisfying each pair and the matrix whose $i, j$ entry is the number of such paths.

TABLE 2

\begin{tabular}{llllll}
\hline \hline Partitions & $f_{i, f_{2}}$ & $f_{1, f_{4}}$ & $f_{2, f_{3}}$ & $f_{3,}, f_{5}$ & $f_{4, f_{5}}$ \\
General Patb & $i j k i j$ & $i j i k j$ & $i k j i j$ & $i k j k j$ & $i k i k j$ \\
Matrix & $M \times M^{2 \prime}$ & $S \times M^{2}$ & $S \times M^{2}$ & $M \cdot S$ & $S \cdot M$ \\
\hline
\end{tabular}

The matrix associated with the pair $f_{1}, f_{2}$ has as its $i, j$ entry the product $m_{i j} m_{i k} m_{k i} m_{i i}$. But since each $m_{i i}$ is 0 or $1, m_{i j} m_{i i}=m_{i j}$. We thus seek the matrix whose $i, j$ entry is $m_{i j} m_{i k} m_{k i}$. But $m_{i k} m_{k i}$ is the $i, j$ entry of $M^{2^{\prime}}$ (the transpose of $M^{2}$ ). Therefore the desired matrix is $M \times M^{2}$.

The matrix associated with the pair $f_{4}, f_{5}$ has as its $i, j$ entry the product $m_{i k} m_{k i} m_{i k} m_{k i}$ which equals $m_{i k} m_{k i} m_{k i}$. But $m_{i k} m_{k i}$ is the $i, k$ entry of $S$, $m_{k i}$ is the $k, j$ entry of $M$ and summation is understood with respect to $k$. Therefore the desired matrix is $S \cdot M$.

All other derivations of matrices associatcd with consistent sets of partitions are similar and will be omitted. 
Thus

$$
R_{4}^{(2)}=M \times M^{2}+S \times M^{2}+S \times M^{2}+[M \cdot S+S \cdot M] .
$$

But $R_{4}=R_{4}^{(1)}-R_{4}^{(2)}$. Hence

$$
\begin{aligned}
R_{4} & =\left[M \cdot d\left(M^{3}\right)+d\left(M^{3}\right) \cdot M\right]+\left[M^{2} \cdot d\left(M^{2}\right)+d\left(M^{2}\right) \cdot M^{2}\right] \\
& +M \cdot d\left(M^{2}\right) \cdot M-M \times M^{2}-2 S \times M^{2}-[M \cdot S+S \cdot M] .
\end{aligned}
$$

The computation of these matrix formulas is considerably simplified by utilizing the following notion of duality: If $a+b+c$ is a partition of $s$, its dual is the partition $c+b+a$. Thus in the partitions of $4, f_{1}$ and $f_{2}$ are duals, $f_{3}$ and $f_{4}$ are duals, and $f_{5}$ is self-dual. Since the dual of a partition is obtained by writing it in reverse order, there is a "reverse order" procedure for determining the matrix associated with the dual of a partition from the matrix associated with the partition itself, namely reverse the order of ordinary matrix multiplication $(\cdot)$ wherever it occurs and leave all other operations unchanged. The reason for this is that the general path corresponding to the dual of a partition is obtained from the original path by interchanging the letters $i$ and $j$ and then reading the original path backwards. Thus, in the formula for $R_{4}^{(2)}$, since the partitions $f_{3}$ and $f_{4}$ are dual, and $f_{8}$ is self-dual, the consistent pairs $f_{3}, f_{3}$ and $f_{4}, f_{5}$ are dual. The duality of the general paths and matrix formulas may be seen by reference to these partition pairs in Table 2.

In formulas (3) and (4), those terms which are duals of each other are enclosed in brackets.

\section{Redundant Paths of Five and Six Steps}

\subsection{Derivation of $R_{5}$.}

The partitions of 5 are

$$
\begin{array}{lll}
f_{1}: 140 & f_{2}: 041 \\
f_{3}: 230 & f_{4}: 032 \\
f_{5}: 320 & f_{8}: 023 \\
f_{7}: 122 & f_{8}: 221 \\
& \multicolumn{2}{l}{f_{9}: 131}
\end{array}
$$

As in the preceding section, consistency tables are constructed for these nine partitions taken 2,3,4,5, and 6 at a time. (By Remark 1 of Section 2, 6 is the greatest possible number of simultaneously consistent partitions of 5.) Also as above, the matrices corresponding to the appropriate general paths are found and substituted into the formula (the special case of equation

(2) for $s=5$ ), 


$$
\begin{aligned}
R_{5}= & R_{5}^{(1)}-R_{5}^{(2)}+R_{5}^{(3)}-R_{5}^{(4)}+R_{5}^{(5)}-R_{s}^{(6)}, \text { the terms of which are: } \\
R_{5}^{(1)}= & {\left[M \cdot d\left(M^{4}\right)+d\left(M^{4}\right) \cdot M\right]+\left[M^{2} \cdot d\left(M^{3}\right)+d\left(M^{3}\right) \cdot M^{2}\right] } \\
& \quad+\left[M^{3} \cdot d\left(M^{2}\right)+d\left(M^{2}\right) \cdot M^{3}\right]+\left[M \cdot d\left(M^{2}\right) \cdot M^{2}\right. \\
& \left.\quad+M^{2} \cdot d\left(M^{2}\right) \cdot M\right]+M \cdot d\left(M^{3}\right) \cdot M, \\
R_{5}^{(2)}= & 3\left[M \cdot d\left(M^{2}\right)^{2}+d\left(M^{2}\right)^{2} \cdot M\right]+\left[M \cdot d\left(M \cdot d\left(M^{2}\right) \cdot M\right)\right. \\
& \left.+d\left(M \cdot d\left(M^{2}\right) \cdot M\right) \cdot M\right]+2\left[M \cdot\left(S \times M^{2}\right)+\left(S \times M^{2}\right) \cdot M\right] \\
& +\left[M \cdot\left(M \times M^{2}\right)+\left(M \times M^{2}\right) \cdot M\right]+\left[M^{2} \cdot S+S \cdot M^{2}\right] \\
& +M \times M^{3}+2 M \times M^{2} \times M^{2}+2 M^{3} \times S \\
& +M^{\prime} \times M^{2} \times M^{2}+d\left(M^{2}\right) \cdot M \cdot d\left(M^{2}\right)+M \cdot S \cdot M, \\
R_{5}^{(3)}= & 6\left[d\left(M^{2}\right) \cdot S+S \cdot d\left(M^{2}\right)\right]+3\left[M \cdot d\left(M^{2}\right)+d\left(M^{2}\right) \cdot M\right] \\
& +\left[M \cdot d\left(M^{2}\right)^{2}+d\left(M^{2}\right)^{2} \cdot M\right]+M^{2} \times M^{\prime}+3 M \times S^{2}, \\
R_{5}^{(4)}= & 9 S+2\left[S \cdot d\left(M^{2}\right)+d\left(M^{2}\right) \cdot S\right]+\left[M \cdot d\left(M^{2}\right)+d\left(M^{2}\right) \cdot M\right], \\
R_{5}^{(5)}= & 6 S, \quad \text { and } \\
R_{5}^{(8)}= & S .
\end{aligned}
$$

Combining these terms, we get

$$
\begin{aligned}
R_{5}=[ & \left.M \cdot d\left(M^{4}\right)+d\left(M^{4}\right) \cdot M\right]+\left[M^{2} \cdot d\left(M^{3}\right)+d\left(M^{3}\right) \cdot M^{2}\right] \\
& +\left[M^{3} \cdot d\left(M^{2}\right)+d\left(M^{2}\right) \cdot M^{3}\right]+\left[M \cdot d\left(M^{2}\right) \cdot M^{2}\right. \\
& \left.+M^{2} \cdot d\left(M^{2}\right) \cdot M\right]+2\left[M \cdot d\left(M^{2}\right)+d\left(M^{2}\right) \cdot M\right] \\
& +4\left[d\left(M^{2}\right) \cdot S+S \cdot d\left(M^{2}\right)\right]+M \cdot d\left(M^{3}\right) \cdot M+M^{2} \times M^{\prime} \\
& +3 M \times S^{2}-\left[M \cdot d\left(M \cdot d\left(M^{2}\right) \cdot M\right)+d\left(M \cdot d\left(M^{2}\right) \cdot M\right) \cdot M\right] \\
& -2\left[M \cdot\left(S \times M^{2}\right)+\left(S \times M^{2}\right) \cdot M\right]-\left[S \cdot M^{2}+M^{2} \cdot S\right] \\
& -2\left[M \cdot d\left(M^{2}\right)^{2}+d\left(M^{2}\right)^{2} \cdot M\right]-\left[M \cdot\left(M \times M^{2}\right)+\left(M \times M^{2}\right) \cdot M\right] \\
& -M \times M^{3 \prime}-2 S \times M^{3}-d\left(M^{2}\right) \cdot M \cdot d\left(M^{2}\right)-M^{\prime} \times M^{2} \times M^{2} \\
& -M \cdot S \cdot M-2 M \times M^{2} \times M^{2 \prime}-4 S .
\end{aligned}
$$

In this equation, as in the preceding equations (3) and (4), those terms which are duals of each other are enclosed in brackets.

\subsection{Derivation of $R_{6}$.}

By a similar procedure the formula for the number of redundant sixstep paths is found. The partitions of 6 are: 


$$
\begin{aligned}
& f_{1}: 150 \quad f_{2}: 051 \\
& f_{3}: 240 \quad f_{4}: 042 \\
& f_{5}: 330 \quad f_{6}: 033 \\
& f_{7}: 420 \quad f_{8}: 024 \\
& f_{9}: 123 \quad f_{10}: 321 \\
& f_{11}: 132 \quad f_{12}: 231 \\
& f_{13}: 141 \quad f_{14}: 222
\end{aligned}
$$

Since the greatest possible number of partitions that are simultaneously consistent is 6 , the formula for $R_{8}$ is found from

$$
R_{8}=R_{6}^{(1)}-R_{8}^{(2)}+R_{6}^{(3)}-R_{6}^{(4)}+R_{6}^{(5)}-R_{6}^{(8)} .
$$

Because of the length of this formula, it is presented in combined form only.

$$
\begin{aligned}
R_{6}=[ & \left.M \cdot d\left(M^{5}\right)+d\left(M^{5}\right) \cdot M\right]+\left[M^{2} \cdot d\left(M^{4}\right)+d\left(M^{4}\right) \cdot M^{2}\right] \\
& +\left[M^{3} \cdot d\left(M^{3}\right)+d\left(M^{3}\right) \cdot M^{3}\right]+\left[M^{4} \cdot d\left(M^{2}\right)+d\left(M^{2}\right) \cdot M^{4}\right] \\
& +\left[M \cdot d\left(M^{2}\right) \cdot M^{3}+M^{3} \cdot d\left(M^{2}\right) \cdot M\right]+\left[M \cdot d\left(M^{3}\right) \cdot M^{2}\right. \\
& \left.+M^{2} \cdot d\left(M^{3}\right) \cdot M\right]+M \cdot d\left(M^{4}\right) \cdot M+M^{2} \cdot d\left(M^{2}\right) \cdot M^{2} \\
& +2\left[M^{2} \cdot d\left(M^{2}\right)+d\left(M^{2}\right) \cdot M^{2}\right]+4\left[M \cdot S \cdot d\left(M^{2}\right)+d\left(M^{2}\right) \cdot S \cdot M\right] \\
& +4\left[M \cdot d\left(M^{2}\right) \cdot S+S \cdot d\left(M^{2}\right) \cdot M\right]+[M \cdot d(M \cdot S \cdot M) \\
& +d(M \cdot S \cdot M) \cdot M]+2 M \cdot d\left(M^{2}\right) \cdot M+4\left[M \cdot d\left(S \cdot M^{2}\right)\right. \\
& \left.+d\left(M^{2} \cdot S\right) \cdot M\right]+4\left[M \cdot d\left(M^{2} \cdot S\right)+d\left(S \cdot M^{2}\right) \cdot M\right] \\
& +\left[M \cdot\left(M^{\prime} \times M^{2}\right)+\left(M^{\prime} \times M^{2}\right) \cdot M\right]+3\left[M \cdot\left(M \times S^{2}\right)\right. \\
& \left.+\left(M \times S^{2}\right) \cdot M\right]+3 M \times\left[S \cdot\left(M^{\prime} \times M^{2}\right)+\left(M^{\prime} \times M^{2}\right) \cdot S\right] \\
& +3 M \times\left[S \cdot\left(M \times M^{2}\right)+\left(M \times M^{2}\right) \cdot S\right] \\
& +M \times\left(M^{\prime} \cdot d\left(M^{2}\right) \cdot M^{\prime}\right)+3 M^{2} \times S^{2}+M^{2} \times M^{2} \cdot \\
& +2 M^{\prime} \times\left[M \cdot\left(M \times M^{2}\right)+\left(M \times M^{2}\right) \cdot M\right]+\left[S \cdot M \cdot d\left(M^{2}\right)\right. \\
& \left.+d\left(M^{2}\right) \cdot M \cdot S\right]+8\left[\left(S \times M^{2}\right) \cdot d\left(M^{2}\right)+d\left(M^{2}\right) \cdot\left(S \times M^{2}\right)\right] \\
& +4\left[\left(M \times M^{2}\right) \cdot d\left(M^{2}\right)+d\left(M^{2}\right) \cdot\left(M \times M^{2}\right)\right]+4\left[S \cdot d\left(M^{3}\right)\right. \\
& \left.+d\left(M^{3}\right) \cdot S\right]+2 S \times\left(M \cdot d\left(M^{2}\right) \cdot M\right)-\left[M^{3} \cdot S+S \cdot M^{3}\right] \\
& -\left[M^{2} \cdot S \cdot M+M \cdot S \cdot M^{2}\right]-\left[M^{2} \cdot\left(M \times M^{2}\right)\right. \\
& \left.+\left(M \times M^{2}\right) \cdot M^{2}\right]-2\left[M^{2} \cdot\left(S \times M^{2}\right)+\left(S \times M^{2}\right) \cdot M^{2}\right]
\end{aligned}
$$




$$
\begin{aligned}
& -2\left[M^{2} \cdot d\left(M^{2}\right)^{2}+d\left(M^{2}\right)^{2} \cdot M^{2}\right]-\left[\left(M \cdot d\left(M^{2}\right)\right)^{2}+\left(d\left(M^{2}\right) \cdot M\right)^{2}\right] \\
& -2 M \cdot d\left(M^{2}\right)^{2} \cdot M-\left[M \cdot\left(M \times M^{3^{\prime}}\right)+\left(M \times M^{3}\right) \cdot M\right] \\
& -2\left[M \cdot\left(M \times M^{2} \times M^{2^{\prime}}\right)+\left(M \times M^{2} \times M^{2}\right) \cdot M\right] \\
& -\left[M \cdot\left(M^{\prime} \times M^{2} \times M^{2}\right)+\left(M^{\prime} \times M^{2} \times M^{2}\right) \cdot M\right] \\
& -2\left[M \cdot\left(S \times M^{3}\right)+\left(S \times M^{3}\right) \cdot M\right]-M \cdot\left(M \times M^{2}\right) \cdot M \\
& -2 M \cdot\left(S \times M^{2}\right) \cdot M-4[M \cdot S+S \cdot M] \\
& -\left[M \cdot d\left(M^{2} \cdot d\left(M^{2}\right) \cdot M\right)+d\left(M \cdot d\left(M^{2}\right) \cdot M^{2}\right) \cdot M\right] \\
& -\left[M \cdot d\left(M \cdot d\left(M^{2}\right) \cdot M^{2}\right)+d\left(M^{2} \cdot d\left(M^{2}\right) \cdot M\right) \cdot M\right] \\
& -\left[M \cdot d\left(M \cdot d\left(M^{3}\right) \cdot M\right)+d\left(M \cdot d\left(M^{3}\right) \cdot M\right) \cdot M\right] \\
& -M \cdot d\left(M \cdot d\left(M^{2}\right) \cdot M\right) \cdot M-\left[M^{2} \cdot d\left(M \cdot d\left(M^{2}\right) \cdot M\right)\right. \\
& \left.+d\left(M \cdot d\left(M^{2}\right) \cdot M\right) \cdot M^{2}\right]-4\left[M \cdot\left(d\left(M^{2}\right) \times d\left(M^{3}\right)\right)\right. \\
& \left.+\left(d\left(M^{2}\right) \times d\left(M^{3}\right)\right) \cdot M\right]-\left[d\left(M^{2}\right) \cdot M \cdot d\left(M^{3}\right)\right. \\
& \left.+d\left(M^{3}\right) \cdot M \cdot d\left(M^{2}\right)\right]-d\left(M^{2}\right) \cdot M^{2} \cdot d\left(M^{2}\right)-4 M \times\left[M^{\prime} \cdot S\right. \\
& \left.+S \cdot M^{\prime}\right]-M \times M^{4}-2 M \times M^{2} \times M^{3 \cdot}-2 M \times M^{2} \times M^{3} \\
& -M^{2} \times M^{2} \times M^{2}-2 M^{\prime} \times M^{2} \times M^{3}-8 S \times[M \cdot S+S \cdot M] \\
& -2 S \times M^{4}-12 S \times M^{2}-8 S \times M^{2}-4 S^{2} .
\end{aligned}
$$

\section{Illustrations}

For the sake of simplicity we consider a sociometric matrix $M$ in which each of six persons chooses three other persons in this group:

$$
M=\left\|\begin{array}{llllll}
0 & 1 & 0 & 1 & 1 & 0 \\
1 & 0 & 0 & 1 & 1 & 0 \\
0 & 1 & 0 & 0 & 1 & 1 \\
1 & 0 & 1 & 0 & 1 & 0 \\
1 & 1 & 0 & 1 & 0 & 0 \\
0 & 1 & 1 & 0 & 1 & 0
\end{array}\right\| .
$$

(Thus person 2 communicates with persons 1, 4, and 5, etc.) For this matrix, we find $R_{3}, R_{4}$, and $R_{5}$.

By equation (3), $R_{3}=M \cdot d\left(M^{2}\right)+d\left(M^{2}\right) \cdot M-S$. The matrix $M^{2}=M \cdot M$ is obtained by ordinary matrix multiplication. 


$$
M^{2}=\left\|\begin{array}{llllll}
3 & 1 & 0 & 2 & 2 & 0 \\
2 & 2 & 1 & 2 & 2 & 0 \\
2 & 2 & 1 & 2 & 2 & 0 \\
1 & 3 & 0 & 2 & 2 & 1 \\
2 & 1 & 1 & 2 & 3 & 0 \\
2 & 2 & 0 & 2 & 2 & 1
\end{array}\right\|, \text { whence } d\left(M^{2}\right)=\left\|\begin{array}{llllll}
3 & 0 & 0 & 0 & 0 & 0 \\
0 & 2 & 0 & 0 & 0 & 0 \\
0 & 0 & 1 & 0 & 0 & 0 \\
0 & 0 & 0 & 2 & 0 & 0 \\
0 & 0 & 0 & 0 & 3 & 0 \\
0 & 0 & 0 & 0 & 0 & 1
\end{array}\right\| .
$$

Thus

$$
M \cdot d\left(M^{2}\right)=\left\|\begin{array}{llllll}
0 & 2 & 0 & 2 & 3 & 0 \\
3 & 0 & 0 & 2 & 3 & 0 \\
0 & 2 & 0 & 0 & 3 & 1 \\
3 & 0 & 1 & 0 & 3 & 0 \\
3 & 2 & 0 & 2 & 0 & 0 \\
0 & 2 & 1 & 0 & 3 & 0
\end{array}\right\|, \quad d\left(M^{2}\right) \cdot M=\left\|\begin{array}{llllll}
0 & 3 & 0 & 3 & 3 & 0 \\
2 & 0 & 0 & 2 & 2 & 0 \\
0 & 1 & 0 & 0 & 1 & 1 \\
2 & 0 & 2 & 0 & 2 & 0 \\
3 & 3 & 0 & 3 & 0 & 0 \\
0 & 1 & 1 & 0 & 1 & 0
\end{array}\right\| .
$$

To find $S=M \times M^{\prime}$, we form the elementwise product of $M$ and $M^{\prime}$, getting

$$
S=\left\|\begin{array}{llllll}
0 & 1 & 0 & 1 & 1 & 0 \\
1 & 0 & 0 & 0 & 1 & 0 \\
0 & 0 & 0 & 0 & 0 & 1 \\
1 & 0 & 0 & 0 & 1 & 0 \\
1 & 1 & 0 & 1 & 0 & 0 \\
0 & 0 & 1 & 0 & 0 & 0
\end{array}\right\|
$$

Substituting the last three matrices into formula (3), we obtain

$$
R_{3}=\left\|\begin{array}{llllll}
0 & 4 & 0 & 4 & 5 & 0 \\
4 & 0 & 0 & 4 & 4 & 0 \\
0 & 3 & 0 & 0 & 4 & 1 \\
4 & 0 & 3 & 0 & 4 & 0 \\
5 & 4 & 0 & 4 & 0 & 0 \\
0 & 3 & 2 & 0 & 4 & 0
\end{array}\right\| .
$$


The matrix $P$. of pure, i.e., non-redundant, $s$-step paths, may be obtained from the formula

$$
P_{.}=\left(M^{*}-R_{\imath}\right)-d\left(M^{*}-R_{s}\right) .
$$

In formula (7), $M^{*}-R_{\mathrm{s}}$ is the matrix obtained by subtracting the matrix of redundant $s$-step paths from the matrix of all s-step paths. However the diagonal of $M^{a}$, although consisting entirely of redundant paths from $i$ to $i$, need not be equal to the diagonal of $R_{\mathrm{a}}$; for the consistency tables for partitions were developed for $i$ to $j$ paths with $i \neq j$. Therefore, the term $d\left(M^{*}-R_{s}\right)$ is subtracted in order to assure that $d\left(P_{s}\right)$ will be zero.

For the special case $s=3$, we get

$$
P_{3}=\left(M^{3}-R_{3}\right)-d\left(M^{3}-R_{3}\right) .
$$

But since $R_{3}=M \cdot d\left(M^{2}\right)+d\left(M^{2}\right) \cdot M-S$, it follows at once that $d\left(R_{3}\right)=0$. Hence $P_{3}=M^{3}-R_{3}-d\left(M^{3}\right)$, from which

$$
P_{3}=\left\|\begin{array}{llllll}
0 & 2 & 2 & 2 & 2 & 1 \\
2 & 0 & 2 & 2 & 3 & 1 \\
6 & 2 & 0 & 6 & 3 & 0 \\
3 & 4 & 0 & 0 & 3 & 0 \\
1 & 2 & 2 & 2 & 0 & 1 \\
6 & 2 & 1 & 6 & 3 & 0
\end{array}\right\|
$$

We illustrate the $(2,5)$ entry of $P_{3}$. The totality of all paths from 2 to 5 is $2-4-3-5,2-1-4-5,2-4-1-5,2-1-2-5,2-5-1-5,2-5-2-5$, and $2-5-4-5$. Since there are seven of these paths, of which the last four are redundant, the $(2,5)$ entries of $M^{3}$ and $R_{3}$ are 7 and 4, respectively.

Using formula (4) and working out the details in a similar fashion as for the case $s=3$ above, we get:

$$
R_{4}=\left\|\begin{array}{llllll}
19 & 12 & 5 & 18 & 17 & 0 \\
17 & 10 & 5 & 18 & 18 & 0 \\
11 & 14 & 1 & 10 & 16 & 2 \\
13 & 15 & 6 & 10 & 18 & 3 \\
17 & 13 & 5 & 18 & 19 & 0 \\
11 & 13 & 2 & 10 & 15 & 1
\end{array}\right\| \text {. But since } M^{4}=\left\|\begin{array}{cccccc}
19 & 15 & 7 & 18 & 20 & 2 \\
18 & 16 & 7 & 18 & 20 & 2 \\
18 & 16 & 7 & 18 & 20 & 2 \\
17 & 17 & 6 & 18 & 20 & 3 \\
18 & 15 & 7 & 18 & 21 & 2 \\
18 & 16 & 6 & 18 & 20 & 3
\end{array}\right\|,
$$


we apply formula (7) with $s=4$ to get

$$
P_{4}=\left\|\begin{array}{llllll}
0 & 3 & 2 & 0 & 3 & 2 \\
1 & 0 & 2 & 0 & 2 & 2 \\
7 & 2 & 0 & 8 & 4 & 0 \\
4 & 2 & 0 & 0 & 2 & 0 \\
1 & 2 & 2 & 0 & 0 & 2 \\
7 & 3 & 4 & 8 & 5 & 0
\end{array}\right\|
$$

Proceeding similarly for the case $s=5$, we get from formulas (5) and (7),

$$
R_{3}=\left\|\begin{array}{llllll}
55 & 46 & 20 & 54 & 59 & 5 \\
53 & 48 & 20 & 54 & 60 & 5 \\
51 & 46 & 16 & 50 & 59 & 7 \\
53 & 45 & 21 & 56 & 60 & 6 \\
53 & 47 & 20 & 54 & 67 & 5 \\
49 & 45 & 17 & 50 & 58 & 2
\end{array}\right\|, P_{3}=\left\|\begin{array}{llllll}
0 & 2 & 0 & 0 & 2 & 2 \\
1 & 0 & 0 & 0 & 1 & 2 \\
3 & 1 & 0 & 4 & 2 & 0 \\
2 & 1 & 0 & 0 & 1 & 0 \\
1 & 1 & 0 & 0 & 0 & 2 \\
5 & 2 & 4 & 4 & 3 & 0
\end{array}\right\| .
$$

Finally $P_{\mathrm{b}}=0$, since except for diagonal entries $R_{\mathrm{b}}=M^{\mathrm{b}}$ by the reasoning of the last sentence of Section 2.1. On substituting this particular matrix $M$ into formula (6), we obtained $R_{6}$. The matrix $R_{6}$ thus obtained was identical with $M^{6}$ except on the main diagonal. This provided an empirical check of formula (6).

\section{Remarks on the General Case}

Given any communication matrix $M$, any positive integer $s$, and enough time, one can find $R_{s}$ by the following process:

(a) write all the $q=C(s, 2)-1$ partitions of $s$ :

$$
f_{1}, f_{2}, \ldots, f_{a}
$$

(b) write all of the $u=C\left(\left[\frac{s+1}{2}\right], 2\right)$ consistency tables,

(c) determine all the general paths corresponding to the consistent collections of partitions,

(d) deduce the matrices of these general paths, and

(e) substitute these matrices into formula (2):

$$
R_{s}=R_{a}^{(1)}-R_{a}^{(2)}+R_{a}^{(3)}-\cdots+(-1)^{1+u} R_{a}^{(u)} .
$$

It would appear desirable to have a recursion formula for $R$, i.e., a relationship expressing $R_{\mathrm{s}}$ in terms of those $R_{t}$ for which $t<s$. Although 
several partial results in this direction have been obtained, some of which may be fruitful, we shall not include these attempts here.

\section{REFERENCES}

1. Festinger, Leon. The analysis of sociograms using matrix algebra. Human Relations, $1949,2,153-158$.

2. Katz, Leo. An application of matrix algebra to the study of human relations within organizations. Institute of Statistics, University of North Carolina, Mimeograph Series, 1950.

3. Luce, R. D., and Perry, A. D. A method of matrix analysis of group structure. Psychometrika, 1949, 14, 95-116.

4. Weiss, Marie J. Higher algebra for the undergraduate. Wiley, 1949, pp. 90-144.

Manuscript received $9 / 6 / 51$

Revised manuscript received 11/6/51 